\title{
$\angle S$ Research Square \\ Elbow Dislocation With Irretrievable Rotating-rope Injury of the Forearm: Case Report and Literature Review
}

\section{Jianchuan Wang ( $\sim$ 2573131767@qq.com )}

Affiliated zhongshan hospital of dalian university

\section{Song Qin}

Affiliated Zhongshan Hospital of Dalian University

\section{Tienan Wang}

Affiliated Zhongshan Hospital of Dalian University

\section{Case report}

Keywords: Essex-Lopresti Injury, Interosseous membrane, Upper and lower radioulnar joint, Rotary noose, Cross damage

Posted Date: February 4th, 2021

DOl: https://doi.org/10.21203/rs.3.rs-166963/v1

License: (c) (i) This work is licensed under a Creative Commons Attribution 4.0 International License. Read Full License

Version of Record: A version of this preprint was published at Orthopaedic Surgery on February 22nd, 2022. See the published version at https://doi.org/10.1111/os.13234. 


\section{Abstract}

Concurrent dislocation of the distal radiulnar joint of the proximal elbow and proximal radius fracture with rotatory noose injury medial to the ulnar tuberosity is an extremely rare case. No such injury has been reported in the domestic or international literature. The injury we report is similar to that of the proximal fibula of the ankle locked behind the tibial tuberosity, namely Bosworth's injury. We report a case of 36 male elbow proximal distal radial ulnar radial ulnar joint rotation joints and proximal radial fracture and dislocation bowstring in ulna nodule inside, in the rapid and timely accurate diagnosis under the emergency line open reduction and accurate judgment and treatment of this injury is challenging, early diagnosis and timely treatment can get the best curative effect.

\section{Introduction}

Geiger's rare and Montesian fractures are classically described in forearm injuries, accounting for 3-6\% and $1-2 \%$ of forearm fractures, respectively[1]. A rare injury associated with distal radioulnar joint dislocation of radial head or neck fracture and rupture of the interosseous membrane is known as EssexLopresti injury [2]. Additionally one kind is Leung [3] for the first time in 2002 put forward forearms cross damage, the damage with the characteristics of the upper and lower radial scale joint dislocation or subluxation occurred at the same time can be associated with the radial head or ulna without ipsilateral ulnar styloid process fracture of radial fractures and interosseous membrane integrity, imaging X-ray side foot radius of cross, sometimes on foot radius for a X-ray also performed before and after cross.

We report the simultaneous elbow proximal radial ulnar distal radial joints' joint dislocation and fracture of the radial head and neck at the same time rotate the noose in ulna nodule inside, this damage is different from Essex - Lopresti forearm fracture dislocation cross damage, accurate of the complex are extremely rare lesions for diagnosis and treatment of challenging, easy misdiagnosis and misdiagnosis, lead to persistent pain and wrist instability, early diagnosis and timely treatment can obtain the best results.

\section{Case Report}

A 36-year-old male holding his child after drinking alcohol complained that he lost his center in the process of playing and rotating. At the moment of falling, in order to protect the child in his right hand, he extended his left hand to support the ground, resulting in severe pain and deformity of his left elbow and wrist, and these joints could not move. At the time of the injury, the elbow was flexed and the wrist was in an extended position. The movement and sensation of the fingers were normal. No neurovascular injury was observed. The patient was admitted to the emergency department at a tertiary general hospital near his home, and radiographs of the forearm(Fig.1), including the wrist and elbow, were taken. Radiographs show dislocation of the elbow proximal and distal radioulnar joint dislocation of the radial head and neck fracture of the rotated noose in the ulnar tuberosity posterior to the humerus and humerus radioulnar relationship. The patient was manually reduced by the emergency physician under brachial plexus 
anesthesia in the orthopaedic emergency department of the first visit hospital, but the initial reduction failed. The radioulnar joint of the radioulnar humerus remained in dislocation. The senior director of orthopedic department performed the second reduction under general anesthesia and reexamined the imaging X-ray after anesthesia woke up. The results showed that the dislocation of the elbow was corrected, but the radius position was not restored (Fig.2). The patient was advised to receive further treatment after plaster fixation, but the patient refused and visited our hospital. Emergency CT examination of the elbow was performed. The CT showed a fracture of the radial head and neck, in which the fracture fragment of the head was detached and the rotational noose of the radial head and neck was posterior to the ulnar tubercle (Fig.3).At this time, the effect of anesthesia is ineffective, the patient complained of elbow pain, feeling a foreign body in the struggle. The elbow was already swollen after two repositions and the skin tension was tolerable. Perfect inspection emergency surgery: and, behold, the radial head and neck fractures, radial proximal noose behind the ulna nodules, application of periosteum stripping prying radial proximal, radius of bowstring proximal quickly bounces, view the complete interosseous membrane without break, the entire fracture of the radial head splicing, two hammer is fixed, then reset the radial head with the backbone, the radial capitulum safety placed a locking plate fixation. The ulnar instability was examined, and 2 Kirschner wires were drilled percutaneous to fix the elbow flexion $100^{\circ}$ under closed reduction, and 2 Kirschner wires were drilled percutaneous to fix the ulnar joint. The intraoperative fluoroscopic position was good.

An ultra - elbow and wrist plaster was used to flex the elbow $90^{\circ}$ times, and the wrist was fixed in supination position for 6 weeks (Fig.4). X-ray plain film was reexamined every week to understand the fracture position. Orthopaedic rehabilitation exercise was performed twice a week from 4 weeks later until the rehabilitation exercise was stopped 6 months after the operation.

After 12 months of postoperative follow-up, the patient was symptom-free and radiographs showed fracture healing (Fig. 5). The forearm was pronated and supination $75^{\circ}$. The elbow flexion Angle ranged from 0 to $140^{\circ}$, and the wrist range of motion reached $80^{\circ}$ and $70^{\circ}$, with full return to work without significant limitation of function (Fig. 6).

\section{Discussion}

Elbow dislocation is the second most common dislocation of the upper limb after the glenohumeral joint, with a reported incidence of 5.2 cases per 100,000 population [4].If a fracture occurs in the case of a dislocated elbow, it is marked as a complex elbow dislocation. These injuries usually require a higher level of energy, and studies have shown that open reduction and internal fixation or radial head replacement for elbow fractures and dislocations can achieve the same range of motion and reliable results[5].However, no cases of elbow dislocation combined with radial head and neck fracture proximal radiulnar joint injury combined with proximal radial fracture with rotatory noose on the medial side of the ulnar tubercle have been reported. Thinking of the forearm as a unit rather than as a single structure is crucial to understanding Essex-Lopresti injury. The forearm device includes the proximal radius and ulnar joint, the distal radioulnar joint, and the interosseous membrane. Essex-Lopresti fracture is a complex 
injury in which longitudinal force is transmitted through the wrist to the radial head and, if sufficient force is applied, a continuous injury can occur. It is characterized by dislocation of the distal radioulnar joint (DRUJ) with fracture of the radial head and rupture of the interosseous membrane[6].Typically in EssexLopresti injuries, attention is focused on the radial head injury, so the distal radial ulnar displacement is ignored at the initial examination. If distal radioulnar instability is not adequately recognized and managed, chronic wrist symptoms such as pain and instability may develop[7].A number of different types of Essex-Lopresti injuries have been reported in the literature, accompanied by unilateral or bilateral elbow dislocation of the radial head and neck fracture and distal radial displacement of the interosseous membrane rupture[8].However, there are no reported cases of elbow dislocation with distal and proximal radioulnar joint dislocation with the rotatory noose of the proximal radius behind the ulnar tubercle and the interosseous membrane intact. Cross forearm injuries with simultaneous dislocation or subluxation of the radioulnar joint are associated with radial head or ulnar styloid fractures with intact interosseous membrane, but not with elbow dislocation and rotational noose of the proximal radius. Cross forearm injuries produce a cross - shaped appearance on lateral radiographs, sometimes even on anteroposterior radiographs.

Essex-Lopresti injury lesions are mainly manifested as forearm instability caused by longitudinal cleavage of the interosseous membrane of the forearm. Since people's attention is mainly focused on the fracture of the radial head, the damage of the inferior radioulnar joint and the interosseous membrane is neglected [9].There was also no diagnostic manifestation of Essex-Lopresti lesion in the cross injury, in which the interosseous ligament structure was remarkably intact, including the separation of the forearm bone before and after the displacement of the proximal radius and the acute phase of excessive tenderness and swelling of the forearm. In previous studies, only 7 adult cases with simultaneous dislocation of the radial head and distal radioulnar joint without fracture were recorded [10].Cross injuries highlight the importance of injuries at the other end of the radiulnar joint, which are also easily overlooked, followed by delayed or neglected distal or proximal radiulnar joint dislocations, for which various stabilizing procedures can be performed [11].In a study of 12 patients with acute ELI, Grassmann et al concluded that early reduction and temporary fixation of the radioulnar joint with Kirschner wires resulted in satisfactory interim results [12].

The case we report is completely different from the Essex-Lopresti injury and the cross injury, in which the entire rotation, flexion and extension device of the forearm is damaged. In our case, there was a dislocation of the elbow, an interchange of the ulnar and radial positions, and more importantly, a fracture of the proximal radius similar to the Bosworth fracture of the ankle. No amount of rotational flexion and extension could reduce the proximal radius of the rotated noose. Preoperative nose-like pain in the patient was considered to be caused by the noose of the proximal radius on the medial side of the ulnar tubercle. If the tension of the interosseous membrane was lost, the noose of the proximal radius would not be behind the ulna, and it could be reduced by rotation. Intraoperatively, we also confirmed the integrity of the forearm interosseous membrane. So the purpose of this clinical cases is to improve the emergency department nurses awareness of this rare injury, and explains in the treatment of such patients should be fully aware of the possible before rare situation, perfect the necessary imaging examination, avoid by all 
means is blind restoration, closed reduction and failure don't trial and error, the fracture like Bosworth ankle-joint injury usually cannot be closed reduction, too much try can backfire, also could aggravate the injury, leading to severe complications.

\section{Declarations}

\section{Acknowledgements}

Not applicable

\section{Authors' contributions}

JW completed the experiment and wrote the article. SQ directed the conception or design of the work and did the analysis. TW revised the article and optimized the language and helped the acquisition and interpretation of data. All authors read and approved the final manuscript.

\section{Funding}

No

\section{Availability of data and materials}

The datasets used and/or analyzed during the current study are available from the corresponding author on reasonable request.

\section{Ethics approval and consent to participate}

The study has been approved by the ethics committee of Affiliated zhongshan hospital of dalian university

\section{Consent for publication}

Yes

\section{Competing interests}

There is no conflict of interest

\section{References}

1. Clare DJ,Corley FG,MA W.Ipsilateral combination Monteggia and Galeazzi injuries in an adult patient:a case report.J Orthop Trauma 2002;16:130-134.

2. Jungbluth P,Frangen $\mathrm{TM}$,Arens $\mathrm{S}$,et al.The undiagnosed Essex-Lopresti injury.J Bone Joint Surg Br 2006;88:1629-1633. 
3. Leung YF, Ip SPS, Wong A et al. Isolated dislocation of the radial head, with simultaneous dislocation of proximal and distal radio-ulnar joints without fracture in an adult patient:case report and review of the literature.Injury 2002;33:271-273.

4. Stoneback JW, Owens BD, Sykes J, et al.Incidence of elbow dislocations in the United States population. J Bone Joint Surg Am 2012;94:240-245.

5. Watters TS, Garrigues GE, Ring D,et al.Fixation versus replacement of radial head in terrible triad:Is there a difference in elbow stability and prognosis? Clin Orthop Relat Res 2014;472:2128-2135.

6. Essex-Lopresti P.Fractures of the radial head with distal radio-ulnar dislocation;report of two cases.J Bone Joint Surg Br 1951;33:244-247.

7. Rodriguez-Martin J, Pretell-Mazzini J, Vidal-Bujanda C.Usual pattern of Essex-Lopresti injury with negative plain radiographs of the wrist: a case report and literature review. Hand Surg 2010;15:4145.

8. Hii J,Page M,Prosser A,Bauer S.An uncommon Essex-Lopresti fracture dislocation with radial displacement in distal direction :diagnosis and surgical treatment of a rare case.BMJ 2013;11:130134.

9. Edwards GS Jr,Jupiter JB.Radial head fractures with acute distal radioulnar dislocation. EssexLopresti revisited. Clin Orthop Relat Res 1988 ;234:61-69.

10. Potter M, Wang A. Simultaneous dislocation of the radiocapitellar and distal radioulnar joints without fracture: case report. J Hand Surg Am 2012;37:2502-2505.

11. Kashyap S, Fein L. Surgical correction of recurrent volar dislocation of the distal radioulnar jont. A case report. Clin Orthop 1991;266:185-189.

12. Grassmann JP, Hakimi M, Gehrmann SV, Betsch M, Kropil P, Wild M, Windolf J,Jungbluth P. The treatment of the acute Essex-Lopresti injury. Bone Joint J 2014 ;96:1385-1391.

\section{Figures}




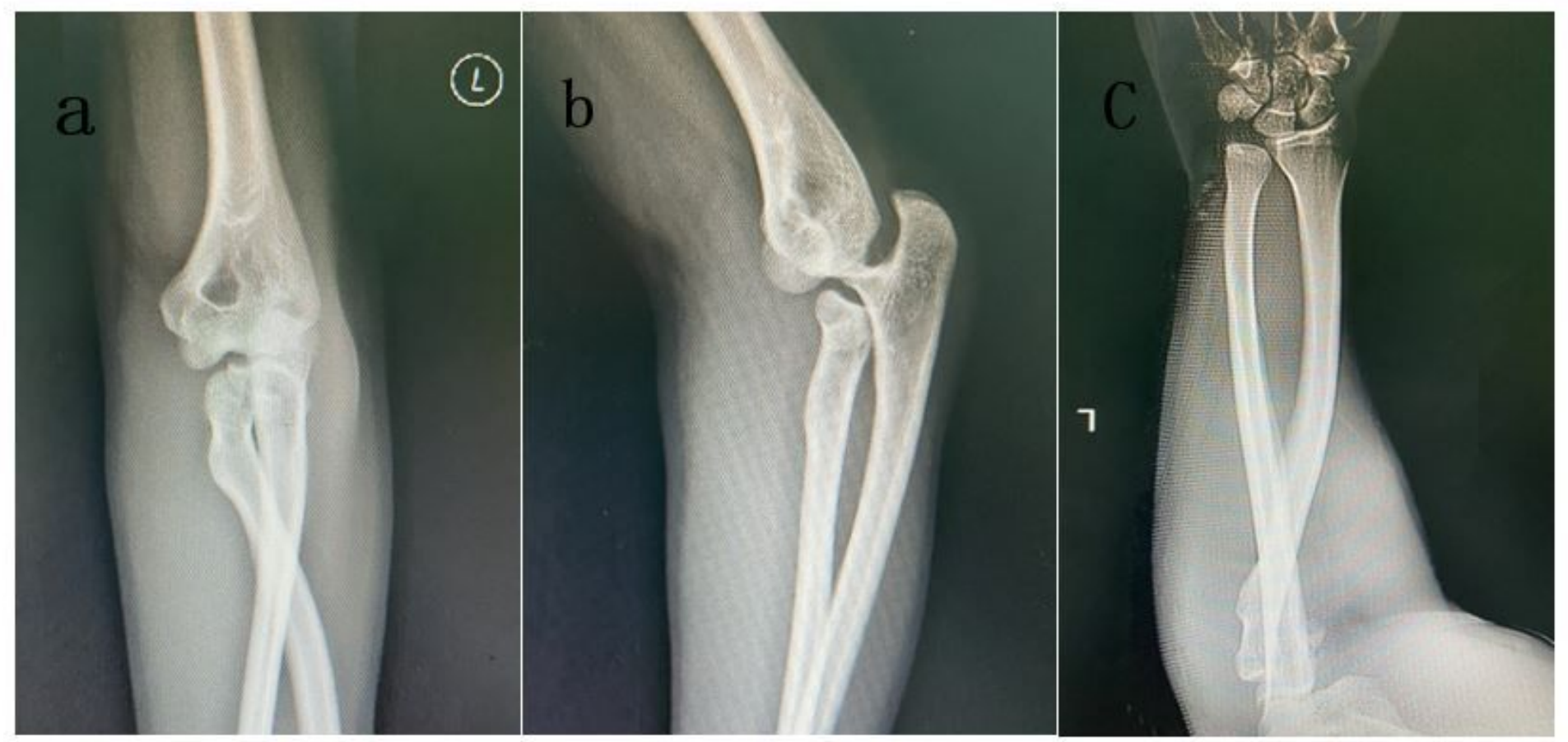

\section{Figure 1}

a Anteroposterior position of the elbow shows the position of the humerulnar humeroceradial relationship, the proximal end of the radius is shifted medially, and the joint with the medial humerocephalus is presented as A cross image. b. Lateral view showing complete dislocation of elbow with intersection of proximal ulna and radius; c Anteroposterior position of the wrist demonstrates distal radioulnar separation and proximal intersecting.

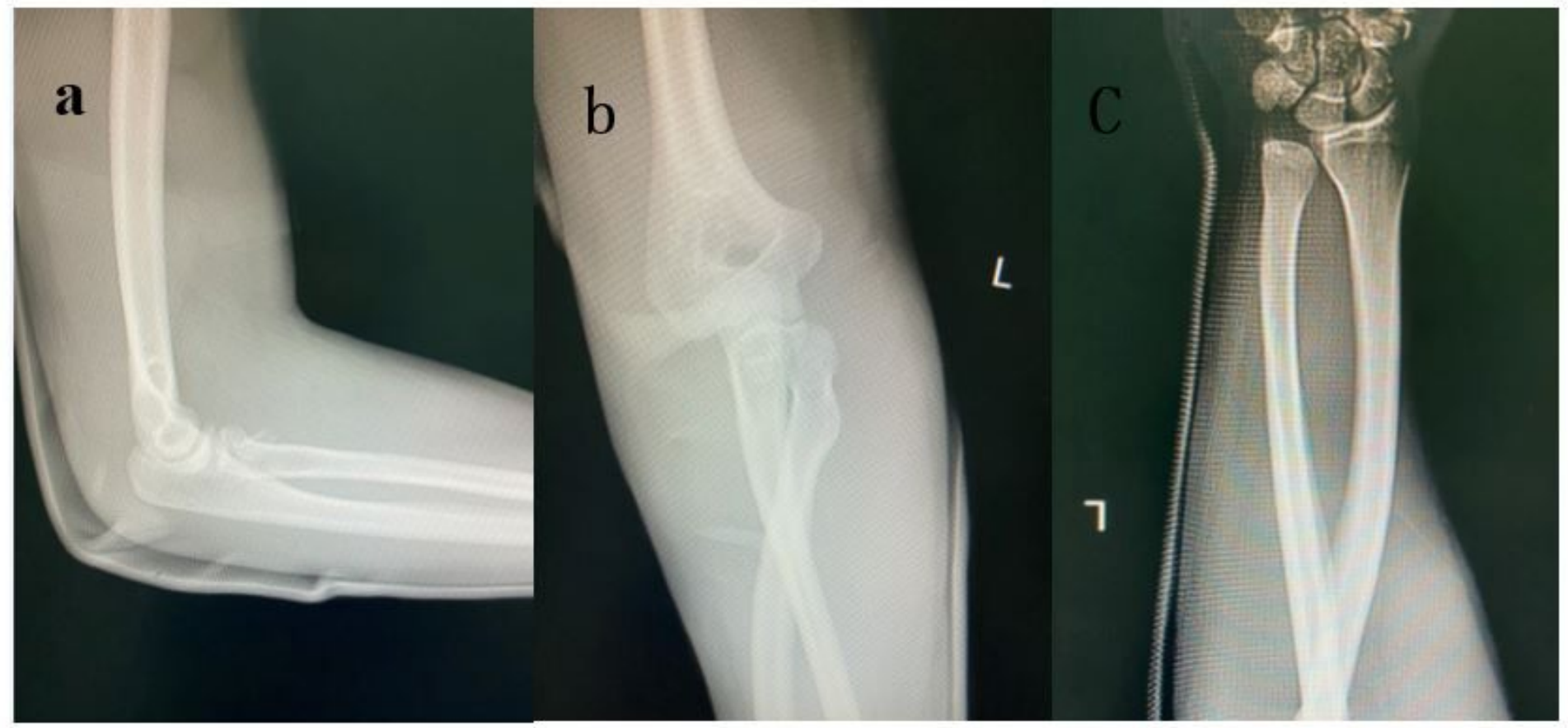

Figure 2 
a Lateral view of the elbow after reduction showed that the ulnar joint returned to normal; b Postreduction anteroposterior radiographs showed recovery of the ulnar joint, asymmetry of the brachioradial joint, and displacement of the proximal radius to the medial side of the ulna; c Anteroposterior position of the wrist showed that the radioulnar joint was still separated and shortened after reduction.

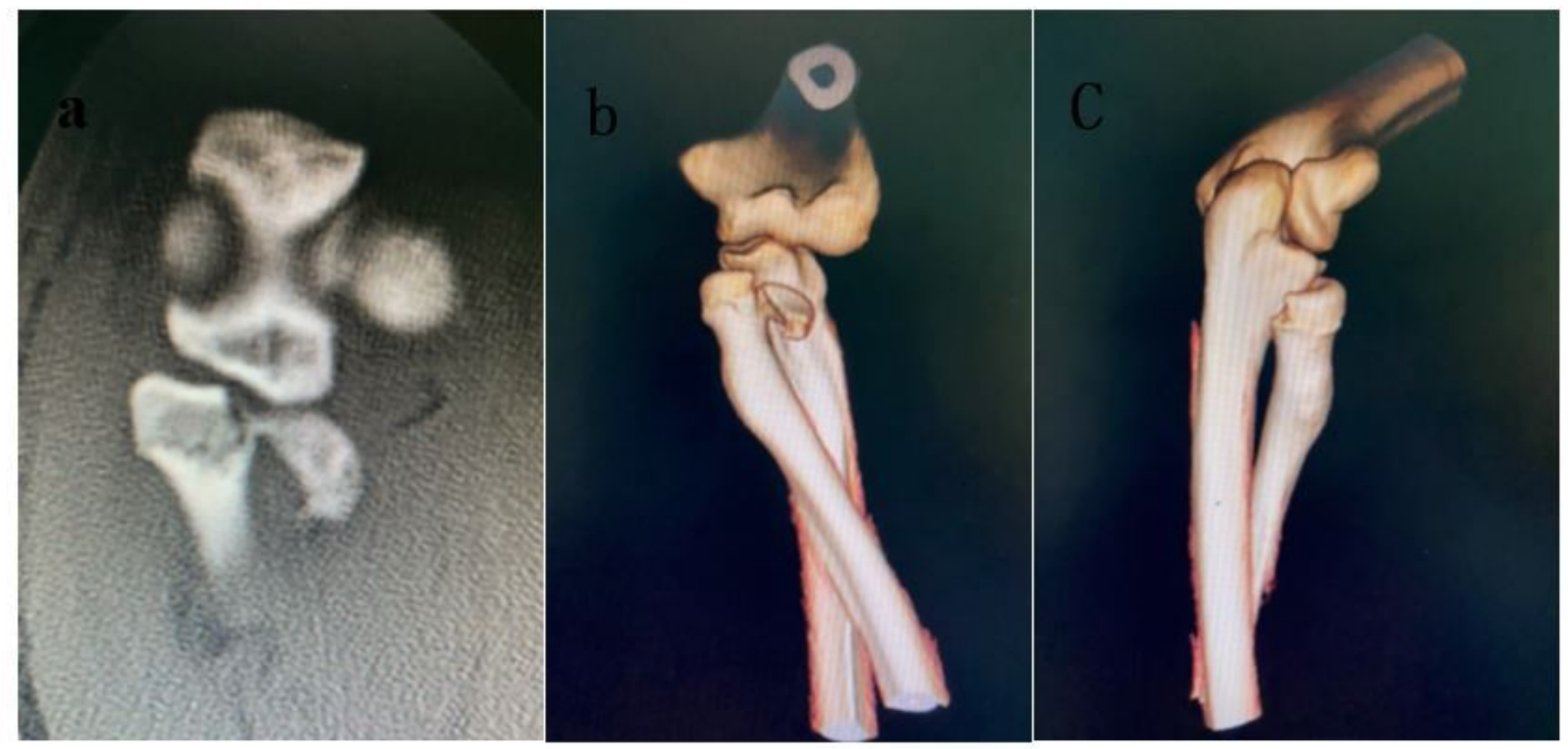

\section{Figure 3}

a Coronal CT showed radial head fracture dislocation and no displacement of radial neck fracture. $b$ Three-dimensional CT of the elbow showed discrete displacement of the radial head but no discrete displacement of the radial neck; $c$ The posterior view of the elbow can more clearly see the radial head rotating the noose to the ulnar tuberosity. 

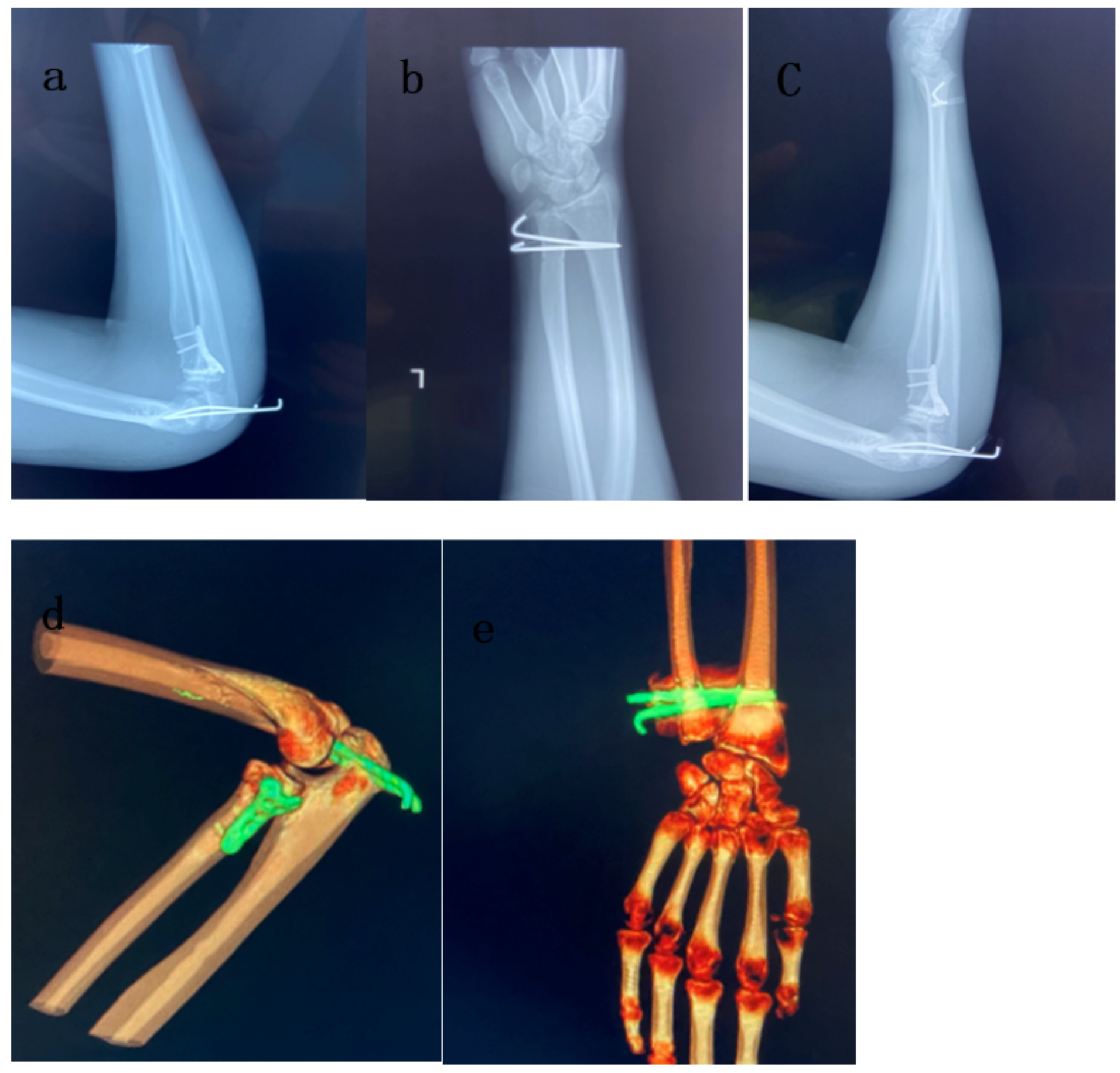

Figure 4

a postoperative elbow showed anatomical reduction of radial head and recovery of ulnar humeroceradial relationship; b. Kirschner wire fixation of distal radiulnar joint and reduction of dislocation; $\mathrm{c} \mathrm{de}$ Postoperative X-ray CT three-dimensional reconstruction of forearm appearance. 


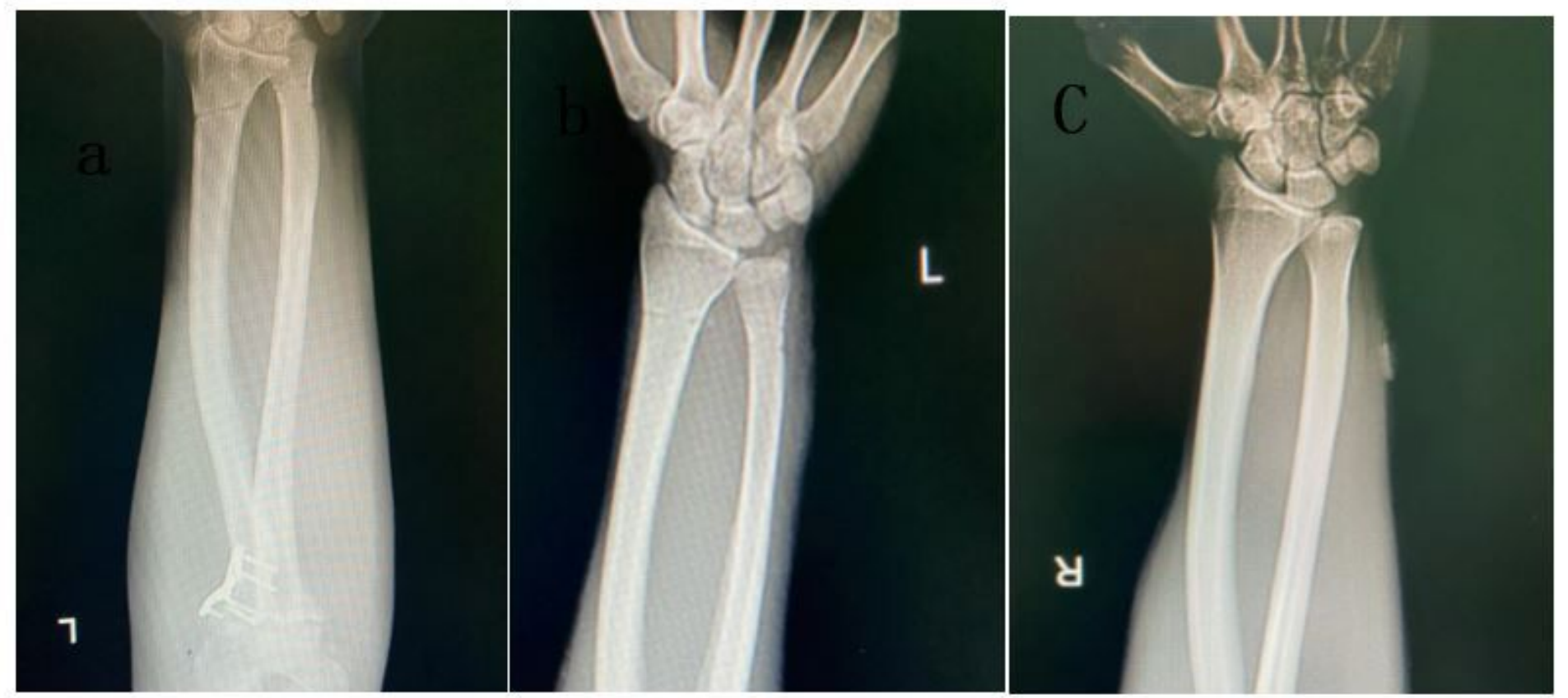

\section{Figure 5}

a postoperative forearm X-ray at 12 months follow-up showed complete fracture healing. b. The distal radiulnar joint was followed up for 12 months without dislocation. c showed no significant difference with the distal radiulnar joint on the healthy side. 

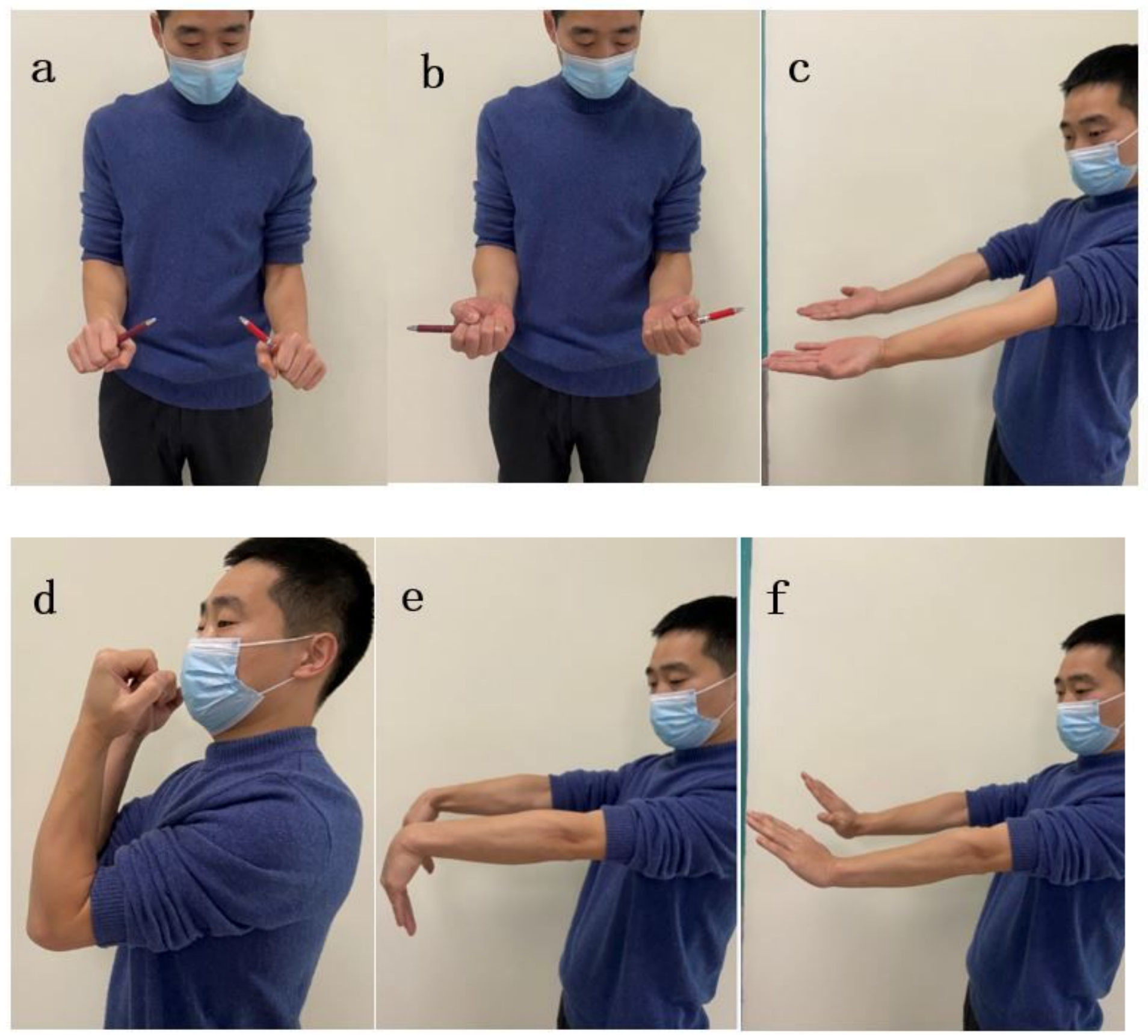

Figure 6

$\mathrm{a} \square \mathrm{b}$ forearm pronation and supination; $\mathrm{c} \square \mathrm{d}$ elbow flexion and extension; $\mathrm{e} \llbracket \mathrm{f}$ wrist flexion and extension. 